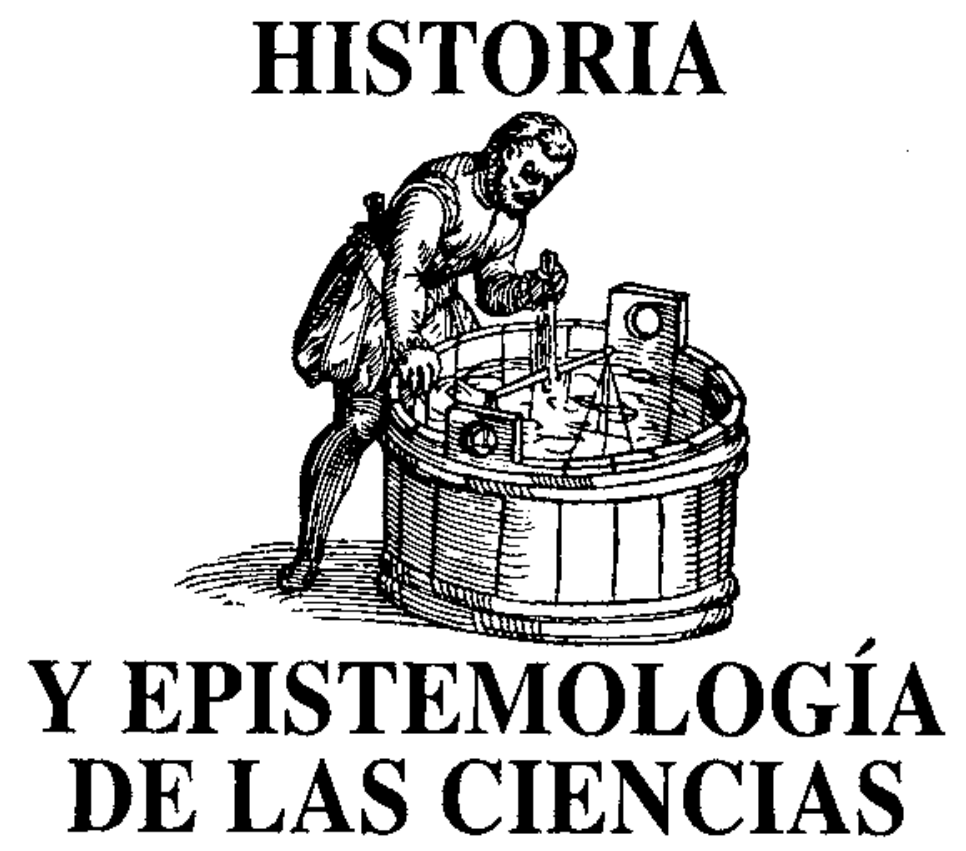

\title{
VINO VIEJO EN BOTELLAS NUEVAS: UN PROBLEMA CON LA EPISTEMOLOGÍA CONSTRUCTIVISTA*
}

\author{
MATTHEWS, M.R. \\ Education Department, Auckland University, Auckland, Nueva Zelanda. \\ * Traducido del inglés por Óscar Barberá y Luis Puig.
}

\section{SUMMARY}

This article deals with what the autor regards as a fatal epistemological error in the constructivist doctrine: its theory maintains the Aristotelian-empiricist epistemological paradigm. However, good constructivist pedagogy could be rescued from the deficient theory that parented it if we made a clear distinction between real and theoretical objects; and knowledge is recognised as a process of intellectual production working with real objects that have been described, apprehended, or incorporated by a theoretical object.

\section{INTRODUCCIÓN}

El constructivismo es una de las influencias principales en la actual didáctica de las ciencias y de las matemáticas. Quizás exagerando, pero sólo levemente, uno de los participantes en el congreso anual de 1991 de la USNational
Association for Research in Science Teaching anunció: ahora todos somos constructivistas. El constructivismo inspira los programas de reforma de enseñanza de la ciencia, infunde animo en los libros de texto, es objeto de 
grandes congresos internacionales y de numerosos artículos de revistas. Sostiene importantes programas de investigación -fuertemente subvencionados- de enseñanza de las ciencias, tales como el proyecto Children's Learning in Science de la Universidad de Leeds (Driver $1978,1983,1989$ ), un proyecto similar en la Universidad de Waikato (Osborne y Freyberg 1985) y el proyecto Student's Intuitions and Scientific Instruction en la Universidad de British Columbia (Kuhn y Aguirre 1987).

El constructivismo también influencia y es inftuenciado por los prolíficos estudios sobre concepciones erróneas y esquemas alternativos en el aprendizaje de las ciencias. En el primer congreso internacional sobre este tema en 1983 se presentaron sesenta trabajos (Helm y Novak 1983) en el segundo congreso se presentaron ciento sesenta (Novak 1987). Estos estudios investigan el pensamiento «científico" de los niños y las conceptualizáciones intuitivas de los fenómenos naturales; también muestran las formas en que las ideas adultas o científicas se incorporan al pensamiento de los niños. Pueden encontrarse resenas de estos trabajos en Driver y Easley (1978), Gilbert y Watts (1983) y Pfundt y Duit (1985).

Los métodos de enseñanza constructivistas son ampliamente propugnados (Clement 1987, Driver y Bell 1986, Oldham 1989, Duckworth 1987), y se han depositado grandes esperanzas en ellos. Uno de quienes abogan por ellos dice que: Si la teoria del conocer que construye el constructivismo [...] se adoptase como una hipótesis de trabajo, produciría algunos cambios bastante profundos en la práctica general de la educación (Glasersfeld 1989, p. 135).

Algunos teóricos han recomendado la creación de currículos de ciencias basados en las líneas constructivistas (Driver y Oldham 1986). El borrador del currículo nacional de ciencias de Nueva Zelanda ha sido elaborado de esta forma. La introducción a este documento dice: este programa pretende ayudar a los estudiantes de diferentes culturas a aprender ciencias en el contexto de sus propias experiencias culturales, sus propias preocupaciones, conocimientos previos, costumbres y lenguaje (Bell 1990, p. 4), y su definición de ciencia es: la exploración e investigación por la gente de sus mundos biológicos, físicos y tecnológicos, haciendo que tengan sentido de manera lógica y creativa. La preferencia del documento por «hacer que tengan sentido» más que por «encontrar la verdad sobre ellos» es característica de la pedagogía y elaboración del currículo constructivistas'.

No ha sido menos prominente el constructivismo en la didáctica de las matemáticas. Un prestigioso escritor ha dicho: Cualquiera que siga la didáctica de las matemáticas ha de estar impresionado por la repentina erupción del aconstructivismo" como preocupación central de tantos investigadores (Davis 1990, p. 104). Un vistazo a las actas de los congresos anuales del International Group for the Psychology of Mathematics Education (PME) confirmará esta observación, como también lo hará una ojeada a las páginas de la mayoria de las revistas sobre didáctica de las matemáticas. Al igual que en la didáctica de las ciencias, el constructivismo ha ofrecido explicaciones de la epistemología de las mate- máticas, del aprendizaje de las matemáticas, de las estrategias de la enseñanza y de la elaboración del currículo. Algunos de estos artículos se reseñan en Kilpatrick (1987) y Leman (1989). El US National Council of Teachers of Mathematics ha publicado recientemente una colección de doce trabajos sobre el constructivismo en didáctica de las matemáticas (Davis, Maher y Noddings 1990; Nel Noddings proporciona en el capítulo inicial un repaso de las raíces intelectuales del constructivismo y su influencia en la didáctica de las matemáticas).

Hay mucho de loable, profundo y progresista en la teoría y práctica constructivista. Es, con mucho, superior a la teoría conductista de la mente y el aprendizajc-espesa y limitada- que Piaget y los primeros psicólogos cognitivos como Bruner combatieron. Su hincapié en que el alumno participe en el aprendizaje y su hincapié en la importancia de comprender los esquemas conceptuales de los estudiantes para poder enseñar de forma fructífera son progresistas y suponen una mejora importante de la enseñanza inspirada en el estímulo-respuesta-refuerzo skinneriano y su paralelo entusiasmo por enunciar los objetivos en términos de conducta. También es progresista el hincapié que se hace en el diálogo, la conversación, la discusión y la justificación de las opiniones del estudiante y el profesor en un contexto social. $\mathrm{De}$ forma importante, el hincapié que el constructivismo hacc en la comprensión como objetivo de la enseñanza es un avance esencial y humano frente al aprendizaje maquinal y la repetición letaníaca de fórmulas que caracterizan tantas aulas de ciencias -aulas como la de universitarios de la cual Richard Feynman dijo: «los estudiantes to han memorizado todo, pero no han entendido el significado de nada» (Feynman 1985, p. 212). Esta aproximación a la enseñanza tipo Gradgrind" se ha extendido desesperanzadoramente en las aulas de ciencias.

Se ha de mencionar que uno no ha de ser un constructivista para estar de acuerdo con la mayor parte de sus propuestas pedagógicas - la enseñanza por Sócrates del teorema de Pitágoras al muchacho esclavo ${ }^{b}$ es un temprano y perdurable modelo de cnseñanza por comprensión-; Aquino y los medievales pusieron énfasis en la necesidad del compromiso personal con la materia que estásiendo enseñada, hablaban de un «amor al aprendizaje»; los ensayos de Montaigne sobre educación abogan por un enfoque dialéctico en lugar de uno didáctico. Dewey y los progresistas hicieron hincapié en la discusión en clase, el debate, la comprobación y escrutinio de las opiniones del profesor y el alumno, tal y como propone también, por supuesto, Paulo Freire en sus muchas publicaciones. Recientemente, Richard Peters, Paul Hirst, Isracl Scheffler, Jane Martin y otros filósofos de la educación han señalado la importancia de la comprensión y el entendimiento del alumno en la enseñanza $a^{2}$. Los más importantes constructivistas han reconocido la existencia previa de una pedagogía buena y progresista que ha precedido sus escritos ${ }^{3}$.

Los aspectos positivos del constructivismo han sido ampliamente desarrollados por otros; este trabajo tratará de resaltar lo que yoconsidero como un error epistemológico fundamental, y fatal, en la doctrina; al resaltar este error 
intento rescatar la buena pedagogía constructivista de la deficiente teoría que la engendró.

En pocas palabras, mi crítica consiste en que el constructivismo conserva el paradigma epistemológico aristotélico-empirista -ampliamente extendido, basado en el de sentido común y centrado en el sujeto-, y que, al apuntar correctamente un importante error en las presunciones empiristas, cambia hacia una epistemología relativista sin abandonar el paradigma mismo. La conclusión relativista sólo se sigue dentro del mismo paradigma empirista; si se rechaza este paradigma -y hay buenas razones para hacerlo- no se siguen tales conclusiones epistemológicas relativistas y, ciertamente, no se siguen conclusiones ontológicas idealistas. Epistemológicamente, el constructivismo es el famoso viejo lobo empirista vestido de oveja contemporánea. Para cambiar la metáfora, es el vino empirista, tan criticado por los constructivistas, servido en botellas nuevas.

\section{FILOSOFÍA CONSTRUCTIVISTA}

El constructivismo es un movimiento heterogéneo. Se han identificado por lo menos las siguientes variantes: contextual, dialéctica, empírica, procesadora de información, metodológica, moderada, piagetiana, postepistemológica, pragmática, radical, realista, social y sociohistórica. A esta lista debe añadirse la variante humanista (Cheung y Taylor 1991), además de la débil y la trivial (Lerman 1989).

El constructivismo está erizado de cuestiones filosóficas: de forma explícita asume posiciones en la filosofía de la ciencia, la filosofía de la mente y la fúlosofía de la educación. Esto no es sorprendente. Es al mismo tiempo una teoría de la ciencia, del aprendizaje humano y de la enseñanza; y existe también un elemento ético cuando los constructivistas escriben sobre la buena enseñanza y educación. El constructivismo hace afirmaciones epistemológicas sobre nuestro conocimiento del mundo y con frecuencia hace afirmaciones ontológicas sobre la naturaleza de ese mundo.

Algunos extractos de escritos constructivistas pueden dar una idea del alcance de estas posiciones epistemológicas y ontológicas:

Tales enfoques constructivistas contemplan el conocimiento como construido personal y socialmente, más que como "objetivo" y revelado; y las teorias, como provisionales, no absolutas (Miller y Driver 1987, p. 57)

La ciencia como conocimiento es un constructo intelectual, y aquello a lo que se llama leyes de la naturaleza son simplemente el resultado de esta actividad humana. La Naturaleza como tal no tiene leyes (Nadeau y Desautels 1984, p. 19).

El hecho de que el conocimiento cientifico nos permita arreglárnoslas bastante bien no justifica la creencia en que el conocimiento cientifico proporciona una imagen del mundo que corresponde a una realidad absoluta (Glasersfeld 1989, p. 35).

Aunque podemos asumir la existencia de un mundo externo, no tenemos acceso directo a él; la ciencia como conocimiento público no es tanto un descubrimiento como una construcción cuidadosamente comprobada (Driver y Oldham 1986, p. 109).

Las ideas y teorías científicas no sólo resultan de la interacción de los individuos con los fenómenos, sino que pasan también por un complejo proceso que implica comunicación y comprobación por parte de importantes instituciones sociales de la ciencia antes de servalidadas por la comunidad científica (Driver 1989, p. 85).

Dicho en términos sencillos, el constructivismo puede ser descrito en esencia como una teoría sobre los límites del conocimiento humano, una creencia en que todo conocimiento es necesariamente un producto de nuestros propios actos cognitivos. No podemos tener un conocimiento directo o sin mediación de ninguna rea. lidad externa uobjetiva. Construimosnuestra comprensión por medio de nuestras experiencias, y el carácter de nuestra experiencia está profundamente influenciado por nuestra lente cognitiva (Confrey 1990, p. 108).

El conocimiento científico se inventa para dar sentido a observaciones que están ellas mismas cargadas de teoría. No existe un gran libro de la naturaleza que puede ser consultado para comprobar si los modelos o las teorías corresponden a una realidad ontológica (Desautels y Larochelle 1990, p. 236).

El constructivismo es, lógicamente, una postura postepistemológica. Las preguntas típicas de epistemología no pueden ser contestadas -ni siquiera formuladas ra. zonablemente- desde esta perspectiva. Sus premisas sugieren, más bien, el abandono del lenguaje epistemo. lógico tradicional (Noddings 1990, p. 18).

La epistemología, incluso cuando se abandona, es importante para el constructivismo, ya que conduce realmente al resto de la teoría y la práctica educacional constructivista. Los constructivistas adoptan la mayor parte de las tesis epistemológicas de la filosofía postpositivista de la ciencia, tesis que han sido muy bien resumidas en Garrison (1986).

Aunque la mayor parte de los constructivistas son realis tas ontológicos - el mundo, aunque no se pueda conocer existe realmente aparte de nuestro pensamiento sobre él--, algunos «constructivistas radicales» son idealistas -el mundo se crea por el pensamiento humano y depende de tal pensamiento-. El constructivismo radical de Ernst von Glasersfeld es quizás el más conocido: el realista cree que sus constructos son una réplica o reflejo de estructuras que existen independientemente, mientras que el constructivista permanece consciente del papel del que experimenta como ente que origina todas las estructuras [...] para el constructivista no hay más estructuras que aquéllas que el que conoce constituye por su misma actividad de coordinación de las partículas de experiencia (Glasersfeld 1987, p. 104). 
Es verdad que su idealismo es muy difícil de concretar. En un cierto momento llega a decir que lectores superficiales o emocionalmente confusos de literatura constructivista han interpretado frecuentemente esta posición como negación de "la realidad» (Glasersfeld 1991, p. xv), pero inmediatamente después de rechazar esta interpretación continúa diciendo: el hecho de que nosotros estemos de acuerdo sobre ciertas cosas y de que podamos comunicamos no prueba que lo que experimentamos tenga realidad objetiva en sí mismo. Si dos personas o incluso toda una sociedad miran a través de lentes distorsionantes y coinciden en lo que ven, ello no hace más real lo que ven -esto sólo significa que sobre la base de tales acuerdos pueden construir un consenso en ciertas areas de sus mundos de experiencias subjetivas.

Grayson Wheatley es un idealista aún más ambiguo, pero su idealismo brilla más claramente en un cierto punto cuando, al estilo de Berkeley, dice: Desde una perspectiva constructivista, el conocimiento se origina en la actividad del aprendiz realizada sobre objetos. Pero los objetos no están desperdigados por el mundo ya elaborado, sino que son constructos mentales (Wheatley 1991, p. 10).

Aunque hay variantes en la epistemología constructivista, la linea común es que está centrada en el sujeto, basada en la experiencia, y es relativista. Pero su relativismo necesita ser distinguido de otros relativismos en los que se acepta como objetivo de la ciencia la búsqueda de la verdad sobre el mundo, y entonces se asevera que entre diferentes explicaciones no podemos conocer cuál es realmente verdadera o mejor. En contraste, para la mayor parte de los constructivistas, nuestro conocimiento no nos informa en absoluto acerca del mundo, nos informa de nuestras experiencias y de cómo están mejor organizadas.

Lerman (1989), siguiendo a Kilpatrick (1987), sugiere que las tesis epistemológicas centrales del constructjvismo son: 1) EI conocimiento es activamente construido por el sujeto cognitivo, no recibido pasivamente del entorno. 2) Llegar a conocer es un proceso de adaptación que organiza el mundo de experiencias del individuo; no descubre un mundo independiente, preexistente fuera de la mente del que conoce.

La primera tesis es una afirmación psicológica, mientras que la segunda es una afirmación epistemológica (como se indicará más adelante, Lerman cree que implica a. Wheatley ofrece un resumen casi idéntico del núcleo epistemológico del constructivismo: La teoría del constructivismo descansa sobre dos principios primordiales El [...] primer principio enuncia que el conocimiento no se recibe de forma pasiva, sino que se construye de forma activa por el sujeto cognitivo [...] El segundo principio enuncia que la función de cognición es adap. tativa y sirve a la organización del mundo experiencial, no al descubrimiento de la realidad ontológica [...] Así, no encontramos la verdad sino que construimos explicaciones viables para nuestras experiencias (Wheatley 1991 , p. 10).

\section{ELCONSTRUCTIVISMO COMOUNA FORMA DE EMPIRISMO}

Dada la influencia del constructivismo, es sorprendente que aparezcan en la literatura sobre cducación pocos críticos de su epistemología. Strike (1987) proporcionó una crítica temprana; ha habido ciertas escaramuzas entre los constructivistas personales de la linea principal piagetiana y los constructivistas sociales vygotskianos. Y ha habido algunos ajustes sobre los límites epistemológicos, por ejemplo por parte de Kilpatrick (1987), Lerman (1989) y Goldin (1990), pero todos ellos pueden considerarse como disputas internas. Es como si un período de ciencia normal kuhniana hubiera descendido sobre las comunidades de didáctica de las ciencias y de las matemáticas. El presidente de la US National Association for Research in Science Teaching sugiere otro tanto: Parece que ahora está ocurriendo una unificación de pensamiento, investigación, desarrollo curricular y educación del profesorado bajo el tema del constructivismo [... hay una] faltade debate polarizado (Yeany 1991 , p. 1).

Un profundo artículo de Wallis Suchting (1992) puede resultar el zorro en el gallinero: su crítica golpea los fundamentos del constructivismo y localiza la doctrina en una antigua tradición filosófica anticientífica ${ }^{4}$.

Para comprender mi argumentación es necesario recorrer de una forma muy esquemática la historia de la epistemología que desde el siglo iv aC ha sido dominada, de una u otra forma, por el paradigma empírico-aristotélico. Aristóteles asumió un mundo independiente de la gente, poblado por sustancias individuales que formaban clases o especies en virtud de sus esencias oformas. Conocer algo era conocer su esencia o naturaleza. En la teoría aristotélica del conocimiento, aquellos que conocen eran pasivos en dos importantes sentidos: 1) el mundo graba conocimientoempíricoen el sujeto a través de la percepción sensorial corriente no mediada; ésta tenía que ser no mediada para que el objeto que se pretendía conocer fuera conocido tal como realmente era; 2) la facultad racional (nous), por un proceso de inducción (o epagoge) intuía las formas universales inherentes en lo real. Como Dewey y Popper han observado, Aristótcles sostuvo básicamente una teoría-«Espectador»-del conocimiento empirica, individualista, de reflejo o de correspondencia: el conocimiento era algo generado por el observador y residente en él. La esencia de los objetos se mostraba en su movimiento natural; el movimiento alterado era el resultado de la interferencia con el natural y, así, no sólo no era de interés para la ciencia, sino además era un obstáculo para ella. En la medida en que el mundo o el objeto se interfería con el conocimiento, éste se frustraba; en la medida en que el sujeto era activo en la adquisición de conocimiento, el conocimiento se ponía en peligro.

La observación, la visión y la contempiación eran los elementos clave en la parte del sujeto de la ecuación epistemológica de Aristóteles, mientras que en la otra parte, la del objeto, el elemento clave cra la naturaleza imperturbada. El conocimiento se obtenía cuando la parte del sujeto reflejaba con precisión especular la parte 
del objeto. El dictum epistemológico de Aristóteles era: decir de lo que es que es, y de lo que no es que no es, es verdadero. Con los escolásticos esto se convierte en la doctrina de que la verdad es la adecuación (o correspondencia) de lás cosas y el intelecto.

A pesar de su retórica antiaristotélica, los empiristas británicos del siglo xvII -Bacon, Locke, Berkeley y Humemantienen en toda su esencia este paradigma aristotélico del conocimiento. El conocisniento era un asunto individual, estaba basado en la experiencia o las impresiones de los sentidos, su criterio era la adecuación entre el pensamiento y la realidad o la correspondencia de las ideas con los objetos.

Cualquier epistemología que formule el problema del conocimiento en términos de un sujeto que observa un objeto y se pregunta hasta qué punto lo que ve refleja la naturaleza o la esencia del objeto, es quintaesencialmente aristotélico o, más generalmente, empirista. Los aristotélicos eran realistas directos sobre la percepción, es decir, los objetos de percepción eran cuerpos materiales; los empiristas posteriores fueron en gran parte realistas indirectos, es decir, los objetos de percepción eran impresiones de los sentidos que se suponían generadas por objetos materiales. Locke, un declarado oponente de Aristóteles, expone así este asuntoen sus Ensayo: La mente, en todos sus pensamientos y razonamientos, no tiene otro objeto inmediato sino sus propias ideas, que ella sola contempla o puede contemplar. Variaciones sobre esto se vuelven a producir en las formulaciones constructivistas modernas. Como es conocido, la formulación de Locke del problema del conocimiento fue utilizada por Berkeley para sostener el idealismo y el relativismo. El argumento de Berkeley en su Tratado era simple pero devastador: En cuanto a nuestros sentidos, por ellos obtenemos el conocimiento sólo de nuestras sensaciones, ideas o de esas cosas que son inmediatamente percibidas por los sentidos, llámeselas como se quiera: pero ellos no nos informan de que las cosas existan sin la mente, o sin percibirlas. No es coincidencia que los constructivistas modernos, una vez formulado el problema epistemológico en términos aristotélico-Jockeanos, proporcionen versiones de la ctítica salvaje de Berkeley y terminen en el relativismo y, para los más consistentes, en el idealismo. No es gratuito que von Glasersfeld diga que 1710 fue un año magnífico: el año en que fueron publicados el principal trabajo de Vico y el Tratado de Berkeleys.

Dentro de este paradigma, marco o problemática, la posibilidad del conocimiento se debilitó una vez se señaló que la mente es activa en la cognición, no sólo porque intuye las formas, sino también porque estructura las experiencias que proporcionan los sentidos; y esta posibilidad se evaporó una vez que se afirmó que los objetos inmediatos de la facultad intelectual eran impre. siones de los sentidos más que la propia naturaleza. La naturaleza, en términos de Kant la cosa en sí, se hizo incognoscible, porque nosotros sólo la podemos observar a través de lentes distorsionantes, y no hay ninguna posición privilegiada desde la que podamos comprobar la adecuación o correspondencia del pensamiento con la realidad. Siguiendo a Kant, Piaget y la hueste de filóso- fos postpositivistas como Toulmin, Kuhn, Feyerabend, Rorty y otros, el constructivismo moderno afirma: los individuos son activos en la adquisición del conocimiento, y así el conocimiento no es posible.

La aceptación por el constructivismo de los fundamentos de la problemática epistemológica aristotélica-empírica queda patente cuando: $a$ ) von Glasersfeld habla de $o b$ servar a través de una lente distorsionante y [estar de acuerdo] en lo que se ve, cuando Confrey habla de lente cognitiva; $b$ ) Desautels y Larochelle escriben sobre dar sentido a observaciones que están ellas mismas cargadas de teoría; c) cuando muchos otros han recurrido a este vocabulario de contemplar-ver-observar para plantear el problema del conocimiento; $d$ ) cuando se utiilizan las figuras ambiguas u ocultas de Kuhn o Hanson para establecer hechos sobre la dependencia de la observación de la teoría con la que se observa e) se usa la terminología importada de la gestalt para describir las revoluciones científicas. Tanto si los sujetos están viendo a través de una lente de manera ciara u oscura, la metáfora de mirar a través de una lente señala el compromiso con una teoría empirista del conocimiento.

La argumentación en un único paso que se sigue desde la premisa psicológica la mente es activa en la adquisición de conocimiento hasta la conclusión epistemológica no podemos conocer la realidad es endémica de los escritos constructivistas. Lerman habla en nombre de otros muchos cuando dice de las dos tesis-indicadas anteriormente como 1 y 2 -que las conexiones entre las hipótesis 1 y 2 parecen ser muy fuertes $(1989$, p. 212).

Pero esta conciusión sólo se sigue si se asume que en verdad Aristóteles definió correctamente el problema del conocimiento; esta conclusión sólo se sigue si se aceptan los términos del problema tal como Aristóteles los enunció. Si se rechaza esta asunción, no se puede derivar ninguna de las conclusiones escépticas del constructivismo.

\section{LA REVOLUCIÓN CIENTÍFICO-EPISTE- MOLOGICA}

Aunque aquí se presente de una forma condensada y en gran parte sugerente, usaré el ejemplo de la revolución científica y epistemológica del siglo xvil para ilustrar las limitaciones del empirismo, en general, y del constructivismo, específicamente, para comprender, y no digamos ya para resolver, el problema epistemológico que presenta la ciencia moderna. El ejemplo también permite mostrar algunas de las intuiciones poderosas, pero parciales, del constructivismo.

La revolución científica asociada con Galileo destruyó con acierto el paradigma epistemológico aristotélico. Desgraciadamente los paladines empiristas de la nueva ciencia deI siglo xvir sólo vieron refutada en los trabajos de Gaifleo y Newton la ciencia aristotélica e interpretaron esta revolución científica en términos de la antigua epistemología, diciendo que la nueva ciencia proporcionaba una mejor correspondencia entre pensamiento y realidad, que era más adecuada a la experiencia, que los 
científicos-filósofos eran mejores pensadores que sus predecesores, etc.

El logro de Galileo es hacer la distinción importante entre el objeto teórico de la ciencia -que es un sistema de definiciones, principios, conceptos y relaciones matemáticamente expresadas- y los objetos reales de la ciencia -que son los matcriales, hechos y objetos del mundo que son entendidos, descritos y manipulados, con la adecuada instrumentación y experimentación, por los científicos y otros, con arreglo a los anteriores. Estos objetos teóricos no son ideas lockeanas que se oponen a los cuerpos materiales; tampoco son ideas humeanas que surgen automáticamente de impresiones de los sentidos. La explicación de Galileo sobre movimiento del péndulo ilustra muy bien la diferencia entre Ios objetos tcóricos de la ciencia de Galileo y los objetos reales del mundo, los péndulos, que son el motivo de esta teoría particular.

Al no tener ninguna mitología en su contra, Galileo no se preocupó de observar cuidadosamente el balanceo de los péndulos y a partir de ello derivar su ley del movimiento isocrónico. De hecho, su mecenas aristotélico Guidibaldo del Monte, en esta ocasión oponente, hizo precisamente esto y no es sorprendente que pensase que Galileo estaba fuera de sus cabales cuando formuló su ley. Los péndulos reales de ninguna manera se comportan isocrónicamente -si lo hicieran, una consecuencia inmediata de la ley de Galileo haría de los péndulos móviles perpetuos, cosa que obviamente no son. De hecho, Galileo comenzó con una colección de conceptos de dinámica, con unos medios matemáticos de representación y análisis que trataban con circunstancias abstractas en las que se ignoraban muchas características de los péndulos reales -su color, su masa, etc- y con unas situaciones idealizadas (en las que la lenteja del péndulo, el grave, se consideraba como una masa puntual; en las que no había fricción mecánica en el fuicro ni resistencia del aire en la oscilación; en las que la cuerda no tenía peso y por tanto no amortiguaba el movimiento; etc.). Cuando el objeto real -el péndulo oscilante no perpetwo, no isocrónico, material-era đescrito en términos proporcionados por el objeto teórico, y representado geométricamente, entonces se encontraba en una forma adecuada para el análisis por la ciencia galileana. El resultado fuc la ley de movimiento isocrónico. Del Monte dijo que éste era un mundo sobre el papel, y que la ciencia debería ser sobre el mundo real. Decisivamente, la ciencia de Galileo era sobre el mundo real; él no era un platónico o idealista, pero su ciencia no era inmediata sobre este mundo tal como está naturalmente dispuesto: estaba mediada por el objeto teórico-conceptual que claramente no se correspondía con el mundo real.

La ciencia de Galileo se aplicaba a una situación experimental altamente constreñida, formada sólo sobre el diseño proporcionado por el objeto teórico. Lo real era alterado, es decir refinado y perfeccionado, para corresponderse con to teórico, y no at revés tal y como el aristotélico del Monte y más generalmente los empiristas insistirían en su pretensión de que la teoría debería alinearse con la realidad. Que el objeto teórico no se correspondía con el real estaba fuera de discusión. Pos- teriormente una interacción de la teoría-abandonando, por ejemplo, la visión de Gálileo de que el círculo cra la braquistocrona o curva de descenso más rápido- y un refinamiento experimental condujeron a la mejora de Huyghen de la ley del movimiento del péndulo.

Ver u observar las cosas de forma diferente no era lo importante en el debate entre Galileo y del Monte. Galileo no veía masas puntuales en el extremo de una cuerda sin peso, ni veía movimiento continuo en cl apogeo de la oscilación; él veía exactamente lo que del Monte veía -lámparas en el extremo de cadenas, bolas de plomo en el extremo de un cable, péndulos que se paraban momentáneamente en su cenit y péndulos que se detenían después de dos docenas de oscilaciones, etc.La observación -tanto si dependía de la teoría como si no- apenas tenía importancia en la disputa. Galileo describió las cosas de forma diferente, no las vió de forma diferente. Las nuevas descripciones no llegaron de la observación -después de todo, uno de los más grandes observadores de todos los tiempos, I eonardo da Vinci, había visto muchas veces péndulos sin describirlos de la forma en que lo hizo Galileo-, las descripciones llegaron desde un objeto teórico construido intelectualmente. Una ve $z$ hubo descrito estas relaciones matemáticamente, pudo trabajar sobre su sistema mecánico. Como ha dicho un comentarista: la observación y la experiencia, en el sentido de experiencia bruta, de sentido común, no representó un papel principal -o, si lo tuvo, fue negativo, el papel de obstáculo, en los fundamentos de la ciencia moderna (Koyré 1968, p. 18). (Una explicación más detallada de este debate sobre el péndulo se desarrolla en Matthews 1987 y 1990).

La mecánica de Newton es la cumbre de la revolución científica y representa también una refutación decisiva del entendimiento empirista de la ciencia. Podemos ver que la estructura de la ciencia moderna emerge con el trabajo de Newton de una manera mucho más explícita que con Galileo.

Sus Principia crean el objeto teórico o aparato de ciencia newtoniana que luego se utiliza para describir y comprender el mundo real, pero las descripciones no pretenden ser reflejos de ese mundo. En las definiciones iniciales de sus Principia introduce los conceptos fundamentales de masa, fuerza, cantidad de movimiento y fuerza centrípeta. Luego especifica las relaciones entre estos conceptos en un conjunto de axiomas. El primer axioma tune el concepto de fuerza al cambio de velocidad; el segundo axioma define la magnitud de fuerza como masa por aceleración; el tercero estipula la naturaleza reactiva de la fuerza. Los Principia elaboran después una ciencia de masas puntuales incoloras, moviéndose en un espacio inercial libre de fuerzas de fricción, por definición. No hay correspondencia entre este objeto tcórico y los cuerpos reales coloreados, afectados por la fricción, no inerciales. Newton se da perfecta cuenta de que las bolas de billar, los carruajes y las balas de cañón no son sistemas inerciales. Pero una vez más esta falta de correspondencia entre lo teórico y lo real simplemente no es lo importante; y por sí mismo, en contra del empirismo, este hecho carece de pertinencia epistemológica. 
Newton reconoce, como lo hizo Gatileo, que su sistema ha de ser sobre el mundo real, pero es sobre el mundo real no en el sentido de una descripción de unos Campos Elíseos imperturbados. Su verdad no consiste en una correspondencia entre las masas puntuales inerciales y las bolas de billar que ruedan o los satélites que giran, sino más bien en la capacidad de las primeras para permitir buenas predicciones sobre las manipulaciones e intervenciones en las segundas.

\section{CRÍTICA CONSTRUCTIVA}

El constructivismo toma la correspondencia entre las ideas y la realidad comoel sine qua non del conocimiento. Cuando se muestra que esto es en principio problemático, los constructivistas concluyen que las pretensiones del conocimiento también deben ser en principio problemáticas, si no manifiestamente quiméricas. Pero la correspondencia no tiene ese papel central en las pretensiones del conocimiento. Lo que las teorías de la correspondencia han apreciado correctamente es que la verdad de una afirmación científica, o el valor de una teoría, está determinada finalmente por algo que está más allá de la misma afirmación o la teoría: el hombre propone, y el mundo dispone. Pero el determinante del conocimiento no es la correspondencia. ¿Qué puede significar el decir que un enunciado, una colección de palabras, se corresponde con un estado material de las cosas? Con seguridad no es el estado de las cosas en crudo, material, to que se supone que se corresponde con la afirmación científica, sino más bien un aspecto delimitado, especificado y normalmente idealizado de este estado material de las cosas -por decirlo de nuevo, las masas puntuales incoloras no se corresponden en ningún sentido con bolas de billar coloreadas-. Las teorías de correspondencia se basan en una intuición realista importante, pero más allá de esto son incoherentes. A fortunadamente esto no afectá nuestras pretensiones de conocimiento.

El constructivismo tiene razón al subrayar los aspectos inventivos, humanos, dependientes de la cultura y de la historia, de la creación de los objetos teóricos de la ciencia-pero nada de esto tiene que ver, por sí mismo, con la verdad-. Aún más, aunque los constructivistas subrayan este aspecto creativo de la producción de conocimiento, rara vez extienden el análisis: su modelo de creación es un tipo de modelo personal, de industria casera; es el modelo personal, a lo Robinson Crusoe, de la construcción de conocimiento que deja de lado las necesarias dimensiones sociales y comunitarias de la cognición -las dimensiones que inclinaron a Freire, siguiendo a Hegel, a decir que es el «nosotros pensamos» lo que determina el "yo pienso", y no al contrario.

La idea de la producción teórica es mucho más exuberante de lo que generalmente nos damos cuenta. Marx dijo que la gente crea la historia, pero no la crean como ellos quieren; la crean en circunstancias determinadas sobre las que tienen poco control. Al igual que ocurre con la producción material, la producción teórica también depende: de las materias primas (palabras, conceptos, lenguaje, fórmulas), los medios de producción (sis- temas matemáticos, lógica, dispositivos para el cómputo, instrumentos), Ias relaciones productivas (formas en que la ciencia se organiza y financia, posesión de la propiedad intelectual, disposiciones educativas) $y$, finalmente, el contexto social y legal de la actividad productiva (fuerzas religiosas, presiones económicas, valores culturales). El conocimiento es una forma de producción teórica y puede ser analizado como tal con provecho.

Wheatley, anteriormente citado, acierta parcialmente cuando dice que los objetos de la ciencia no están desperdigados por ahí. Donde él y la mayor parte de los constructivistas, junto con Aristóteles y los empiristas, yerran es al no ser capaces de distinguir los objetos teóricos de la ciencia, que no están desperdigados por ahí, de los objetos reales de la ciencia, que sí están desperdigados por ahí y caen sobre las cabezas de la gente. De forma significativa, hasta los objetos teóricos, una vez ya producidos, tienen una realidad incluso si no se los encuentra desperdigados. La mecánica de Newton, la teoría de la evolución de Darwin y la genética de Mendel existen y pueden ser aprendidos por pensadores posteriores y enseñadas a ellos -simplemente no tienen que ser confundidos con manzanas que caen, animales que pacen o campos de guisantes.

Gran parte de lo que se ha argìido aquí fue enunciado por Marx en la primera de sus tesis sobre Feuerbach en 1844. Allí dijo que el principal defecto del materialismo en su tiempo fue ignorar la dimensión activa y subjetiva (en el sentido de los sujetos humanos) de la producción y adquisición de conocimiento. Marx dijo que esta parte fue desarrollada por el idealismo que, sin embargo, no fue capaz de apreciar la realidad u objetividad de la actividad intelectual humana. EI constructivismo ha hecho casi exactamente lo mismo. (Para una elaboración de la epistemología de Marx véase Matthews 1980 , capítulo 6)

Una vez hecha la distinción entre objeto real y teórico, y reconocido el conocimiento como un proceso de producción intelectual que trabaja con objetos reales que han sido descritos, aprehendidos o incorporados por un objeto teórico, pueden comenzar las interesantes tareas epistemológicas de evaluar diferentes modos de producción de conocimiento -en términos de fecundidad, simplicidad, utilidad-. El relativismo cortocircuita todo esto.

Así también puede comenzar el problema pedagógico de identificar qué dominios teóricos, o discursos, o actividades productivas son válidas y educativamente adecuadas, y embarcar a los estudiantes en la práctica y evaluación de estos dominios.

El empirismo, y el constructivismo, concibe la empresa de la ciencia en términos de individuos que observan el mundo y tratan de comprobar si sus ideas, conceptos y conceptualizaciones tienen sentido. La epistemología objetivista distingue entre la materia prima y los hechos del mundo (los objetos reales de la ciencia), las estructuras teóricas y los conceptos de la ciencia, el material y los hechos como son descritos por la teoría (los objetos teóricos de la ciencia) y los procedimientos experimen- 
tales y técnicos de la ciencia. La comprensión y conceptualización individual es parásita de este dominio científico extra-individual. La didáctica de las ciencias puede ser concebida entonces en términos de la introducción apropiada de los individuos en este mundo de conceptos, comprensiones, técnicas y normas comunitarias.

Von Glasersfeld dice con frecuencia que la problemática epistemológica ortodoxa debe ser abandonada y que la correspondencia como marca de verdad ha de ser rechazada. Esto es así porque en sus términos no podemos ver la realidad, sólo tenemos nuestras sensaciones para reflejarla y, por tanto, nunca podemos juzgar la correspondencia entre nuestras ideas y el mundo (una nueva

\section{NOTAS DE LOS TRADUCTORES}

a Thomas Gradgrind es un personaje de la novela Tiempos dificiles de Charles Dickens (Hard Times. For These Times, 1854. Bradbury \& Evans: London). Se trata de un maestro de escuela caracterizado por una pedagogía basada en el aprendizaje memoristico de hechos, en la que se encuentran ausentes la imaginación, la creatividad, las teorías y, en general, todo aquello no observable.

\section{NOTAS DEL AUTOR}

I Tal como se indicará más adelante, aquí se presenta un dilema para cl constructi vismo: muchas ideas y constelaciones de ideas pueden "tener sentido» para un individuo, y esto no tiene ninguna conexión en absoluto con su veracidad o legitimidad. Los constructivistas apuntan razonablemente que enseñar verdades que simplemente no tienen sentido para los individuos y que permanecen para siempre como un misterio para ellos es dudosamente una buena pedagogía; pero hay una zona intermedia entre esto y la pretensión de que simplemente dar sentido es el objetivo de la educación; y, por supuesto, ello requiere una argumentación enteramente diferente para mostrar que «hacer que tenga sentido" es el objetivo de la ciencia y que es realmente distinto deI de «encontrar la verdad».

2 Neil Cooper es representativo de estos filósofos de la educación al proponer: Mi tesis es que la enseñanza debería ser la transmistón de comprensión-conocimiento y que una persona educada sera la que no solo tenga comprensiónconocimiento, sino que entienda la naturalezade ese conocimiento. (Cooper 1987, p. 61).

3 Ernst von Glasersfeld ha comentado de forma realista que: Los buenos profesores [...] han puesto en práctica gran parte de lo que aquí se sugiere, sin el beneficio de una teoria explicita del conocer [...] pero el constructivismo puede proporcionar a los miles de educadores menos intuitivos una forma accesible de mejorar sus métodos de instrucción. (Glasersfeld 1989, p. 138). exposición del argumento de Berkeley). Él reemplaza a veces la correspondencia con el pragmatismo y, en otros lugares, con la coherencia entre las experiencias. Esto le conduce, al igual que a Noddings por ejemplo, a hablar del constructivismo como postepistemológico. Su formulacion original de un sujeto que observa un objeto le conduce a un callejón sin salida, y entonces se entiende por qué quiere abandonar la epistemología. Mi sugerencia es que éste es un ejemplo de viejo y agrio vino empirista dentro de una botella nueva; existen otros vinos objetivistas, no empiristas, que permiten que el buen beber epistemológico prosiga con interés, disfrute y provecho.

- No tenemos constancia de que Sócrates enseñara nunca a ningún esclavo el teorema de Pitágoras. El autor probablemente se refiere al famoso pasaje $82 b-85$ b del Menón de Platón, pero ahí lo que Sócrates hace es guiar al esclavo para que descubra, o recuerde, cuánto vale o cú́l ha de ser el lado de un cuadrado cuya área sea el doble de otro de cuatro pics, y no enseñarle el teorema de Pitágoras.

4 El artículo de Jeremy Kilpatrick de 1987, leido en la International Psychology of Mathematics Conference polarizo el debate en la comunidad de didáctica de las matemáticas.

5 Que un destacado constructivista identifique a Vicoy Berkeley como los fundadores del constructivismo y alabe su filosofía es índice de la ambigua relación entre el constructivismo y la ciencia moderna, incluyendo la didácica de las cicncias: Vico y Berkeley eran oponentes declarados de la revolución científica y del trabajo de Galileo, Newton y otros, los cuales eran defensores del cristianismo ortodoxo frente a la observada filosofía irreligiosa de la nueva ciencia. El Tratado de Berkeley de 1710 se subtitulaba Donde se indagan las causas principales del error y la dificultad en las ciencias, con base en el escepticismo, el ateísmo y la irreligión. Que sean tantos los que actualmente defienden la didáctica de las ciencias con las doctrinas de dos pensadores tan profundamente anticientíficos debe causar algún tipo de reflexión.

- Para alguna elaboración de epistemología objetivista, no empirista, véase Suchting (1986, cap. 1), Chalmers (1982, caps. 10,11) y Mathews (1980) 


\section{REFERENCIAS BIBLIOGRÁFICAS}

BELL, B., 1990. Draft Forms 1-5 Science Syllabus, Ministry of Education, Wellington.

CHALMERS, A.F., 1982. What Is This Thing Called Science? (University of Queensland Press: St. Lucia).

CHEUNG, K.C. y TAYLOR, R., Towards a Humanistic Constructivist Model of Science Learning: Changing Perspectives and Research Implications, Joumal of Curriculum Studies, 23(1).

CLEMENT, J., 1987. Overcoming Students Misconceptions in Physics: The Role of Anchoring Intuitions and Analogical Validity, en Joseph D. Novak (ed.) Proceedings of the Second International Seminar on Misconceptions \& Educational Strategies in Science \& Mathematics, pp. 84-97. (Education Department Cornell University: Ithaca).

COFREY, J., 1990. What Constructivism Implies for Teaching, en R.B. Davis, C.A. Maher y N. Noddings (eds.), Constructivist Viewss on the Teaching and Learning of Mathematics, pp. 107-122. (National Council of Teachers of Mathematics, Reston, VA).

COOPHR, N., 1987. The Transmission of Knowledge, en R. Staughton y J. Wilson (eds.), Philosophers on Education, pp. 61-78. (Barnes \& Noble: Totowa NJ).

DAVIS, R.B., 1990. Discovery Learning and Constructivism, en R.B.Davis, C.A. Mahery N. Noddings (eds.), Constructivist Views on the Teaching and Learning of Mathematics, pp.93106. (National Council of Teachers of Mathematics, Reston, VA).

DAVIS, R.B., MAHER, C.A. y NODDINGS, N. (eds.), 1990. Constructivist Views on the Teaching and Learning of Mathematics. (National Council of Teachers of Mathematics, Reston, VA).

DESAUTELS, J. y LAROCHELLE, M., 1990. A Constructivist Pedagogical Strategy: The Epistemological Disturbance (Experiment and Preliminary Results) en D.E. Herget (ed.), More History and Philosophy of Science in Science Teaching. (Florida State University, Tallahassee, Florida).

DRIVER, R., 1983. The Pupil as Scientist. (Open University, Milton Keynes).

DRIVER, R, 1989. The Construction of Scientific Knowledge in School Classrooms, en R. Millar (ed.), Doing Science: Images of Science in Science Education, pp. 83-106. (Faimar Press, Lewes, East Sussex).

DRIVER, R. y EASLEY, J, 1978. Pupils \& Paradigms: A Review of Literature Related to Concept Development in Adolescent Science Students, Studies in Science Education, 5, pp. 61-84.

DRIVER, R. y BELL, B., 1985. Students Thinking and the learning of Science: A Constructivist View, School Science Review, 67 (240), pp. 445-456.

DRIVER, R. y OLDHAM, V., I986. A Constructivist Approach to Curriculum Development in Science, Studies in Science Education, 13, pp. 105-122.

DUCKWORTH, E., 1987. The Having of Wonderful Ideas. (Teachers College Press, Columbia University: Nueva York).
FEYNMAN, R.P., 1985. Surely You're Joking, Mr, Feynman. (Allen \& Unwin: Londres).

GARRISON, J.W., 1986, Some Principles of Postpositivist Philosophy of Science, Educational Researcher, 15(9), pp. 12-18.

GLBERT, J.K. y WATTS, D.M., 1983. Concepts, Misconceptions \& Alternative Conceptions: Changing Perspectives in Science Education, Studies in Science Education, 10, pp. 61-98.

GLASERSFELD, E. von, 1989. Cognition, Construction of Knowledge, and Teaching, Synthese, 80(1), pp. 12I-140.

GLASERSFELD, E von, 1991. Introduction, en E.V. Glasersfeld (ed.), Radical Constructivism in Mathematics Education, pp. Xili-Xx. (Kluwer: Dordrecht).

GOLDIN, G., 1990. Epistemology, Constructivism and Discovery Learning in Mathematics, en Davis, R., Maher, C. y Noddings, $\mathrm{N}$. (eds.), Constructivist Views on the Teaching and Learning of Mathematics. (National Council of Teachers of Mathematics, Reston, VA).

HELM, H. y NOVAK, I.D., (eds.), 1983. Proceedings of the International Seminar on Misconceptions in Science \& Mathematics. (Education Department, Cornell University: Ithaca).

HILPATRICK, J., 1987. What Constructivism Might Be in Mathematics Education, en J.C. Bergeron, N. Herscovics y C. Keiran (eds.), Psychology of Mathematics Education, pp. 3-27. Proceedings of the Eleventh International Conference, Montreal.

KOYRÉ, A., I968. Metaphysics and Measurement. (Harvard University Press: Cambridge, MA).

KUHN, K. y AGUIRRE, J., 1987. A Case Study - on the «Journal Method». A method Designed to Enable the Implementation of Constructivist Teaching in the Classroom, en Joseph D. Novak (ed.), Proceedings of the Second International Seminar on Misconceptions \& Educational Strategies in Science \& Mathematics, pp. 262-274. (Education Department, Cornell University: Ithaca).

LERMAN, S., 1989. Constructivism, Mathematics and Mathematics Education, Educational Studies in Mathematics, 20, pp. $211-223$.

MATTHEWS, M.R., 1980. The Marxist Theory of Schooling: A Study of Epistemology and Education. (Harvester Press: Brighton).

MATTHEWS, M.R., 1987. Galileo's Pendulum \& the Objects of Science, en B. y D. Amstine (eds.), Philosophy of Education, po. 309-319, Philosophy of Education Society.

MATTHEWS, M.R., 1990. Galileo and Pendulum Motion: A Case for History and Philosophy in the Science Classroom, The Australian Science Teachers Journal, 36(1), pp. 7-13.

MILLAR, R, y DRIVER, R., 1987. Beyond Processes, Studies in Science Education, 14, pp. 33-62.

NADEAU, R. y DESTAUTELS, J., 1984. Epistemology and the Teaching of Science. (Science Council of Canada: Ottawa). 
NODDINGS, N., 1990. Constructivism in Mathematics Education, en R.B. Davis, C.A. Maher y N. Noddings (eds.), Constructivist Views on the Teaching and Learning of Mathematics, pp. 7 . 18. (National Council of Teachers of Mathematics, Reston, VA).

NOVAK, J.D., (ed). 1987. Proceedings of the Secondlntemational Seminar on Misconceptions \& Educational Strategies in Science \& Mathematics. (Education Department, Cornell University: Ithaca).

OLDHAM, V., 1989. A Constructivist Approach to the Teaching of Plant Nutrition: A Report on a Scheme in Action. (Centre for Science and Mathematics Education, University of Leeds).

OSBORNE, R.J. y FREYBERG, P., 1985. Learning in Science: The Implications of Children's Science. (Heinemann: Londres).
PFUNDT, H. y DUIT, R., 1985. Bibliography of Students Alternative Frameworks \& Science Education, $2^{a}$ ed. (Institute for Science Education, University of Kiel).

STRIKE, K.A., 1987. Towards a Coherent Constructivism, en J.D. Novak (ed.), Misconceptions \& Educational Strategies, Vol. I, pp. 481-489. (Education Department, Cornell University).

SUCHTING, W.A., 1986. Marx and Philosophy. (MacMillan: Londres).

SUCHTING, W.A., 1992. Constructivism Deconstructed, Science \& Education, 1(3), pp. 223-254.

WEATLEY, G.H., 1991. Constructivist Perspectives on Science and Mathematics Learning, Science Education, 75(1), pp. 922.

YEANY, R.H., 1991. A Unifying Theme in Science Education?, NARST News, 33(2), pp. 1-3. 